\section{Pioloform F Instead of Formvar as a Support Film for TEM}

Victoria J. Madden, University of North Carolina - Chapel Hill

Pioloform $F$ (Wacker Chemie Co., Munich, Germany) is an excellent plastic for use as a support film for electron microscopy. The advantages of Pioborm $F$ are high mechanical and thermal stability, and low material bulk (Stockem, 1970). Because of these properties, thinner films than Formuar or collodion may be used and remain stable under the electron beam without subsequent stabilization by carbon coating. Thin support films of gray interference color are prepared by dissolving 0.5 grams of Pioloform $F$ powder in 100 milliliters of anhydrous chloroform (Stockem, 1970), using the dip method described by Reimer (1967). The Pioloform solution can be stored indefinitely in a brown bottle containing molecular sieve; this shortens preparation time and involves less handling of the components which are carcinogenic. Just before use, pipet the neoessary amount of solution from the bottle without disturbing the molecular sieve. Our laboratory routinely uses Pioloform to support single slot grids, unstable ultrathin sections, and as a substrate for negative staining.

Fiolotorm films are similar to Formvar films in that the film surface is hydrophobic and carries a net positive charge. In negative stain techniques, the hydrophobic property of boh films causes uneven spreading of the specimen and the stain. Common methods for rendering hydrophobic support films hydrophilic such as glow discharge, coating with cationic dyes, and the use of surfactants are effective for Pioloform films (tor an excellent review of these methods, see Hayat and Miller, 1990).

A common pitfall is when films such as Pioloform are used in immunocylochemical procedures involving colloidal gold probes. Non-specific background labeling of the film often results from the electrostatic attraction of the

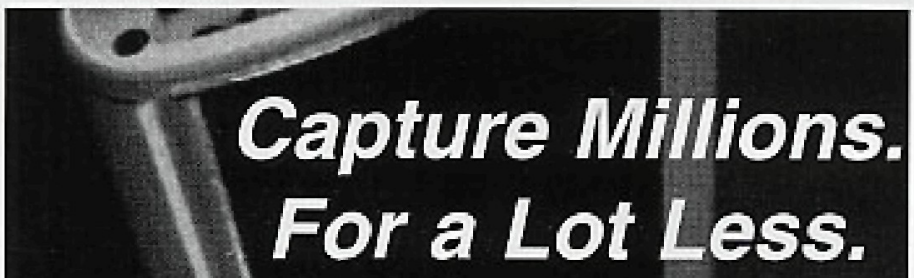

Capture high quality images with the Polaroid Digital Microscope Camera. At $1600 \times 1200$ resolution, the Folaroid DMC has everything you need. Best of all. it's easy to use, easy to afford.

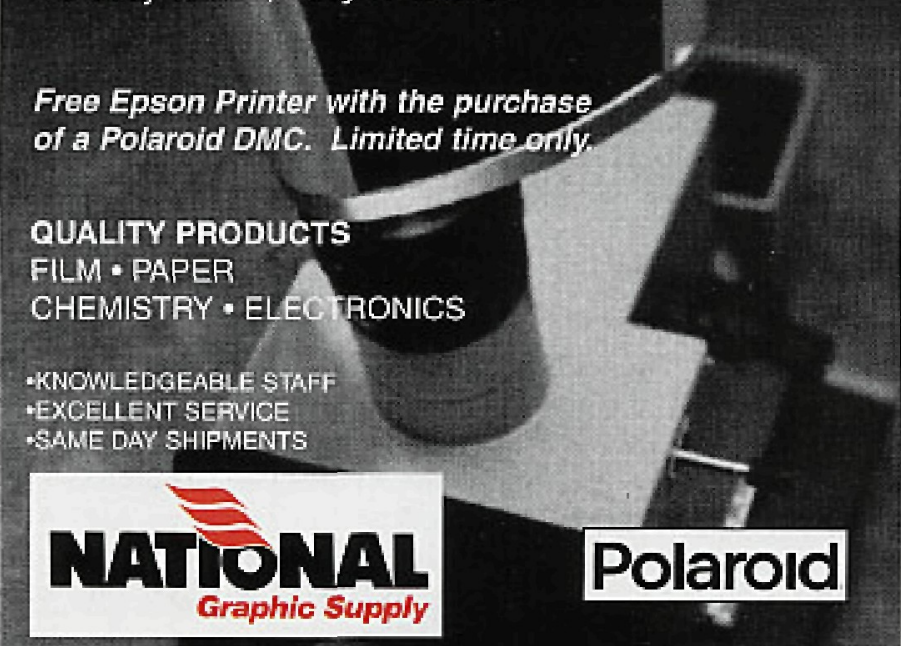

226 North Allen Street, Albany, New York 12206 (600) 223-7130 Fax (800) 832-2205

e-mall reply@ngscorp.com wwwngscopp.com negatively charged regions of the gold particle to the positively charged surface of the film. Using fish gelatin as a protein stabilizer for the immunogold complex (Birrell, 1987) or inoorporation of gelatin (Eloom $\# 60-100,1 \%$ wiv) in the dilution and rinse buffers (Behnke, 1986) can reduce background problems. Other effective remedies are to use surfaclants such as Tween-20 (0.05-0.1\% w/v) in the dilution and rinse buffers or by increasing the salt concentration of the rinse buffer after the immunogold incubation slep (i.e., use $1.0 \mathrm{M}$ Tris-buffered saline instead of $0.1 \mathrm{M}$ ). Typically, we use a combination of several methods to prevent background when using Fioloform-ooated grids with immunogold staining. For $\frac{c}{\bar{v}}$ example, the dilution and rinse buffers usually contain $0.1 \mathrm{M}$ Tris - or phosphate- $\frac{\bar{n}}{\overline{0}}$ buffered saline (at the appropriate $\mathrm{pH}$ for the antibodies), $0.1 \%$ cold-water fish skin gelatin, $0.05 \%$ Tween-20, and for the last two rinses after incubating in immunogold and before post-fixing in glutaraldehyde, rinse in $1.0 \mathrm{M} \mathrm{Tris-HCl}, \mathrm{pH}$ 8.2. As for any immunolabeling procedure, different specimen types and gold probes will require modifying or combining these methods to achieve cptimal results.

\section{References}

Behnke, 0, T. Ammittzboll, H. Jessen, M. Klokker, K. Nilausen, J.

Tranumi-Jensen, L. Olsson. 1986. Non-specific binding of protein-stabilized gold sols as a source of error in immunocytochemistry. Eur. J. Cell. Biol. 41:326.

Birrell, G.E., K.K. Hedberg, and 0.H. Griffith, 1987. Pitfalls of immunogold labeling: Analysis of light microscopy, transmission electron microscopy, and photoelectron microscopy. J. Histocheni. Cytochem. 35: 843-63.

Hayat, M.A. and S.E. Miller, 1990. Negative Staining. MoGraw-Hill Publishing Company, New York, 215-224.

Reimer, L 1967. Eloktronemikroskopische Untersuchungs- und Prapara tionsmelhoden. 2. Aufl. Spring- L'erlag, Berlin.

Stockem, W. 1970. The suitability of Pioloform $F$ for the preparing of electron microscopic supporting films. Mikroskopie 26 (5'6):185-189.

\section{For more contact information:}

Microsoopy Services Laboratory

CB\# 7523 Brinkhous-Bullitt Eldg.

Dept. of Pathology and Laboratory Medicine

University of North Carolina

Chapel Hill, NC. 27599-7525

tel: $919-966-2413$

e-mail: vmadden@med.unc.edu

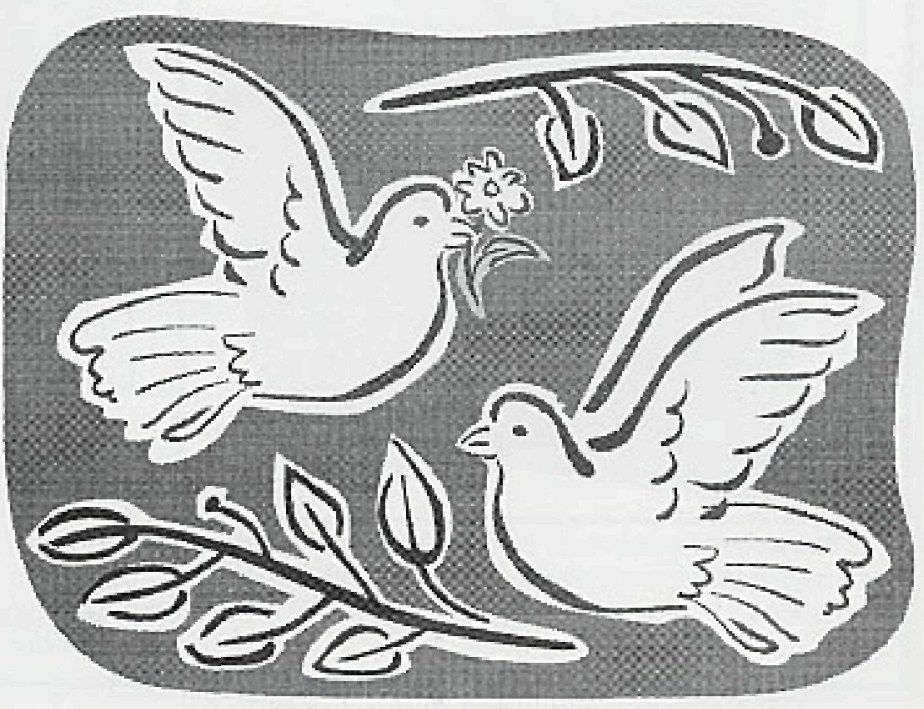


$\gamma \mathbf{P}|\mathbf{G}| \mathbf{T}]$

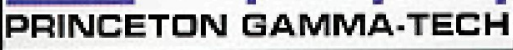
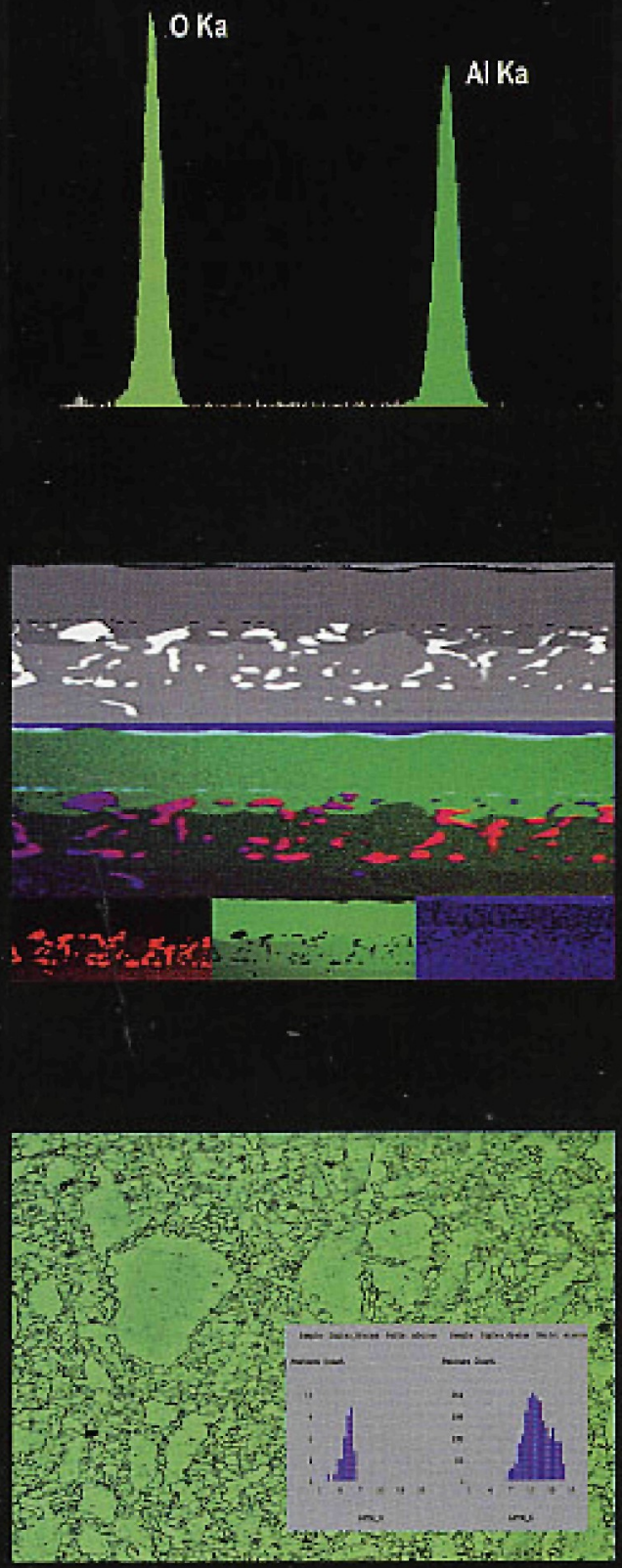

Princeton Gamma-Tech, Inc. C/N 863 Princeton, NJ 08542-0863

Telephone: (609) 924-7310

Facsimile: (609) 924-1729

e-mail: sales.ppgr.com websiter wwwpgt.com

\section{The Best Value In Microanalysis}

\section{Avalon 4000:}

\section{Low cost EDS upgrade!}

\section{Avalon 8000:}

\section{Workhorse for}

\section{everyday solutions!}

\section{IMIX:}

\section{Simply the best!}

For over 30 years you have come to PGT for the latest innovations in X-ray microanalysis and EDS detector technology.

Now you can come to us for inexpensive upgrades, fundaimental workhorse systems, as well as solutions for advanced applications. 